Am J Physiol Heart Circ Physiol. 2007 July ; 293(1): H425-H432. doi:10.1152/ajpheart.01217.2006.

\title{
Cyclooxygenase and nitric oxide synthase dependence of cutaneous reactive hyperemia in humans
}

\author{
Marvin S. Medow ${ }^{1,2}$, Indu Taneja ${ }^{1,3}$, and Julian M. Stewart ${ }^{1,2,3}$ \\ 1'Department of Pediatrics, New York Medical College, Valhalla, New York \\ ${ }^{2}$ Department of Physiology, New York Medical College, Valhalla, New York \\ ${ }^{3}$ Department of Medicine, New York Medical College, Valhalla, New York
}

\begin{abstract}
We tested the hypothesis that cyclooxygenases (COXs) or COX products inhibit nitric oxide (NO) synthesis and thereby mask potential effects of $\mathrm{NO}$ on reactive hyperemia in the cutaneous circulation. We performed laser-Doppler flowmetry (LDF) with intradermal microdialysis in 12 healthy volunteers aged 19-25 yr. LDF was expressed as the percent cutaneous vascular conduction $(\% \mathrm{CVC})$ or as the maximum $\% \mathrm{CVC}\left(\% \mathrm{CVC}_{\max }\right)$ where $\mathrm{CVC}$ is $\mathrm{LDF} /$ mean arterial pressure. We tested the effects of the nonisoform-specific NO synthase inhibitor nitro-L-arginine (NLA, $10 \mathrm{mM}$ ), the nonspecific COX inhibitor ketorolac (Keto, $10 \mathrm{mM}$ ), combined NLA + Keto, and NLA + sodium nitroprusside (SNP, $28 \mathrm{mM}$ ) on baseline and reactive hyperemia flow parameters. We also examined the effects of isoproterenol, a $\beta$-adrenergic agonist that causes prostaglandin-independent vasodilation to correct for the increase in baseline flow caused by Keto. When delivered directly into the intradermal space, Keto greatly augments all aspects of the laserDoppler flow response to reactive hyperemia: peak reactive hyperemic flow increased from $41 \pm 5$ to $77 \pm 7 \% \mathrm{CVC}_{\max }$, time to peak flow increased from $17 \pm 3$ to $56 \pm 24 \mathrm{~s}$, the area under the reactive hyperemic curve increased from $1,417 \pm 326$ to $3,376 \pm 876 \% \mathrm{CVC}_{\max } \cdot \mathrm{s}$, and the time constant for the decay of peak flow increased from $100 \pm 23$ to $821 \pm 311$ s. NLA greatly attenuates the Keto response despite exerting no effects on baseline LDF or on reactive hyperemia when given alone. Low-dose NLA + SNP duplicates the Keto response. Isoproterenol increased baseline and peak reactive flow. These results suggest that COX inhibition unmasks NO dependence of reactive hyperemia in human cutaneous circulation.
\end{abstract}

\section{Keywords}

microdialysis; prostaglandin; skin

\begin{abstract}
Reactive hyperemia (RH) is the sudden rise in muscle and skin blood flow that can be measured after release of ischemic arterial occlusion. It has been used as a clinical tool to evaluate both micro- and macrovascular function in normal subjects, as well as those with various disease states $(11,31,33,34)$. Following release of the occlusion, postischemic
\end{abstract}

Address for reprint requests and other correspondence: J. Stewart, The Center for Pediatric Hypotension, New York Medical College, 19 Bradhurst Ave. Ste. 3050N, Hawthorne, NY 10532 (stewart@ nymc.edu). 
vasodilation occurs in most tissues (30), but the precise vasodilatory response differs from one vascular bed to another. RH has been studied most often in skeletal muscle vasculature where it is currently thought that hyperemia is due to a combination of the myogenic response (4) and local vasoactive tissue-related substances, notably prostaglandins (PGs). Other factors that appear to strongly relate to the peak flow of the hyperemic response are the local concentration of potassium (17), nitric oxide (NO) (24), adenosine (9), and endothelium-dependent hyperpolarizing factor (EDHF) (37), which have been proposed as potential mediators for the excess cumulative blood flow, i.e., the total amount of blood delivered in excess of that absent during ischemia (19). Thus RH in muscle relates to bioavailable NO, local vasodilator substances, PGs, and EDHF.

RH has also been studied in skin (30); however, cutaneous RH response could be different from RH in skeletal muscle and in other tissues. Thus, whereas NO contributes to RH in most other tissues $(11,13,15,24,37)$, its contribution to cutaneous RH appears to be minimal $(35,38)$. For example, Wong et al. (35) have shown that the duration of the falloff in flow of the cutaneous circulation is independent of NO synthase (NOS) inhibition, whereas Zhao et al. (38) provided data that $\mathrm{NO}$ concentration measured by amperometric electrode did not change during RH. Also, the role of PGs in RH has been controversial (12, 20). Thus whereas Larkin and Williams (20) studying laser-Doppler measurements of skin blood flow showed that $\mathrm{RH}$ in the human forearm is mediated by a local reflex involving sensory nerves and a cyclooxygenase (COX) product, Dalle-Ave et al. (12) showed that there was no significant response to acute systemic COX inhibitors. However, the systemic administration of drugs does not always produce an adequate blockade in the skin $(28,29)$.

NO, NOS isoforms, and COX products mutually interact since there is increasing evidence suggesting considerable "cross talk" between NO and PG biosynthetic pathways involving an active back modulation operated by reaction end products, including NO, PGs, and cyclic nucleotides (22). For example, thromboxane $\mathrm{A}_{2}$ exerts inhibitory effects on bioavailable NO through increasing oxidative stress (32) and inhibiting NOS (36). We hypothesized that COX or COX products mask NO effects on RH by inhibiting NOS. We therefore performed experiments that were designed to investigate the interactive contributions of COX and NOS inhibition and of NO replacement to cutaneous RH.

\section{METHODS}

\section{Subjects}

We recruited 12 healthy volunteers (7 men and 5 women) aged 19-25 yr (mean, $22.6 \pm 0.4$ yr). Only subjects found to be free from systemic diseases and cardiovascular disease were eligible. Subjects did not partake of any medications and refrained from alcohol and caffeinated beverages for $24 \mathrm{~h}$ before the study. There were no smokers or trained competitive athletes. Informed consent was obtained, and the Committee for the Protection of Human Subjects (Institutional Review Board) of New York Medical College approved all protocols. Supine heart rate, systolic blood pressure, and mean arterial pressure were not significantly different in females compared with those in males. Female subjects were enrolled without regard to the phase of their menstrual cycle. 
General protocol-Microdialysis catheters were used to infuse drugs locally. Before microdialysis catheter insertion, laser-Doppler flow (LDF) was measured over each of four insertion sites to get an approximation of baseline flows for later use to determine when the area had recovered from catheter insertion. Laser probes were removed, and four microdialysis catheters were inserted. After recovery (see Instrumentation), LDF was measured while the catheters were perfused with lactated Ringer solution, and values were recorded for $10 \mathrm{~min}$. Following this, LDF was recorded during ischemia and RH. To evaluate the influence of biochemical mediators on this response, we produced NOS inhibition using the nonisoform-specific NOS inhibitor nitro-L-arginine (NLA), COX inhibition using the nonselective COX inhibitor ketorolac (Keto), inhibition of both NOS and COX using NLA + Keto, and NO donation during NOS inhibition by perfusing NLA with sodium nitroprusside (SNP). Before the effects of the drugs were tested, lactated Ringer solution was perfused through all the catheters, and the subjects were allowed to recover before the introduction of a drug, which was dissolved in the same vehicle. Thus all determinations were compared with baseline determinations obtained during perfusion of vehicle alone. Thus, in this experimental design (shown in Fig. 1), the baseline values represent a placebo, and because each catheter was perfused with only drug (or combination), washout periods were not needed to obtain the described measurements. Each of these drugs or drug combinations was perfused for $30 \mathrm{~min}$. Following drug administration, a second postdrug baseline was recorded for each microdialysis catheter. Ischemia and $\mathrm{RH}$ were then repeated while drug infusions continued, and the skin response to RH was recorded. After recovery from this second RH and as a final infusion, $28 \mathrm{mM}$ SNP was delivered through each microdialysis catheter to obtain maximum blood flow.

Instrumentation-All testing was conducted in a temperature-controlled room $\left(\sim 25^{\circ} \mathrm{C}\right)$ at least $2 \mathrm{~h}$ after a light breakfast. Measurements were made in the left calf. Since all experiments were performed while the subject remained supine, the leg was at the level of the heart throughout all procedures. Subjects were instrumented with four microdialysis catheters, placed at least $4 \mathrm{~cm}$ apart, inserted in the dermal space of the lateral aspect of the left calf after gentle hair removal from the insertion site. Each probe (MD-2000 Linear Microdialysis Probes, Bioanalytical Systems, West Lafayette, IN) has a 10-mm microdialysis membrane section that is placed in the intradermal space using a 25 -gauge needle as an introducer. Catheters were randomly designated as 1, 2, 3, and 4.

Following placement, all catheters were initially perfused with Ringer solution at $2 \mu \mathrm{l} / \mathrm{min}$. An integrating laser-Doppler flow probe (Probe 413, Perimed, Stockholm, Sweden) containing seven individual probe tips (each contains a separate transmitting and receiving fiber) was then placed directly over each microdialysis catheter to measure skin blood flow, designated as LDF. LDF was then recorded until values were similar to those measured over the same area before catheter insertion. The return of LDF to approximately preinsertion values usually occurred by $60 \mathrm{~min}$ (1), designated as the recovery period. When necessary, longer times were allowed until preinsertion LDF was reached. 
Drug infusions-Once baseline LDF values were obtained and RH was measured under untreated conditions, subjects received perfusate containing $10 \mathrm{mM}$ NLA, $10 \mathrm{mM}$ Keto, 10 $\mathrm{mM}$ NLA $+10 \mathrm{mM}$ Keto, and $10 \mathrm{mM} \mathrm{NLA}+28 \mathrm{mM}$ SNP in catheters $1-4$, respectively, at a rate of $2 \mu \mathrm{l} / \mathrm{min}$. This scheme is shown in Fig. 1. We used NLA instead of $N^{\omega}$-nitro-Larginine methyl ester (L-NAME) because the latter can act as a muscarinic receptor antagonist $(5,8)$. Keto infusion resulted in a significant increase in baseline LDF and an increase in percent cutaneous vascular conduction (\% CVC) to a plateau by $20 \mathrm{~min}$, maintained for $>90 \mathrm{~min}$.

To determine whether this increase in baseline would influence the results of subsequent maneuvers, in separate experiments on another day, we infused isoproterenol $(50-150 \mu \mathrm{M})$ in a dose sufficient to increase the treated baseline to values equivalent to that using Keto. This was done by measuring LDF while Keto was infused through one catheter and by comparing the response to isoproterenol infused through catheters 2 and 3 . The concentration of Keto used was chosen to conform to the concentrations of the drug used in prior work $(16,18,21)$.

$\mathbf{R H}$-To cause RH, an occlusion cuff placed around the midthigh of the left leg was inflated to a pressure of $30 \mathrm{mmHg}$ above the systolic pressure and remained inflated for $5 \mathrm{~min}$, after which it was rapidly released. An absence of cutaneous flow during ischemia was documented by achieving a near-zero LDF as shown in Fig. 2. Peak flow and time to peak flow were measured. The time constant of decay of peak flow was determined by logarithmic transform of the LDF time series, followed by least-squares fit using the singular value algorithm (26). The excess cumulative blood flow-the total amount of blood delivered in excess of that absent during ischemia elicited upon release of arterial occlusion -was recorded until the full recovery of LDF to the basal level. Excess cumulative flow was estimated by the area under the RH curve (AUC) with respect to the final averaged steady-state baseline once recovery was complete, as shown in Fig. 2, middle (right) and bottom. The AUC was computed as follows: the steady-state baseline was subtracted from the signal, and the signal was integrated as a function of time. This resulted in values (shown in Fig. 2, bottom) in which the difference between the minimum and plateau represents the AUC. Data analysis was performed using custom software.

\section{Monitoring}

Heart rate was monitored by electrocardiography, and right upper extremity blood pressures were measured by finger plethysmography intermittently recalibrated against oscillometry in the right arm. Mean arterial pressure was obtained by averaging the signal over 5 min.

\section{Data and Statistical Analysis}

Laser-Doppler skin blood flows were measured in arbitrary perfusion units. Continuous LDF data were collected at a sampling rate of $200 \mathrm{~Hz}$ during experiments. Data from the lasers were multiplexed and interfaced to a personal computer through an analog-to-digital converter (DI-720, DATAQ industries, Milwaukee, WI) using custom data acquisition software. LDF data were converted to units of CVC by dividing by the mean arterial pressure. 
$\mathrm{CVC}$ measurements were then converted to a maximum $\% \mathrm{CVC}\left(\% \mathrm{CVC}_{\max }\right)$ by dividing $\mathrm{CVC}$ by the maximum CVC achieved by the administration of $28 \mathrm{mM} \mathrm{SNP}$ at the end of the experiments. This fraction was converted to a percentile by multiplying by 100 . Conductance data were therefore displayed as $\% \mathrm{CVC}_{\max }$.

Changes in baseline LDF before drugs and after drugs were compared by one-way ANOVA (Fig. 3). Results are shown and reported as means \pm SE. All other comparisons were made by repeated-measures ANOVA to look at differences in RH parameters between pre- and postdrug infusion using the particular microdialysis catheter as the within factor. We compared peak reactive hyperemic flows before and after drug treatment, differences in the peak flows from the treated baseline, and the percent changes in time to peak flow, AUC, and the time constant of decay of peak flow during RH. Results were calculated using SPSS (Statistical Package for the Social Sciences) software version 11.0. The value for a was $<0.05$.

\section{RESULTS}

The responses measured by LDF in the skin to RH were not significantly different when comparing data obtained from men and women. Therefore, male and female data were pooled.

\section{Effects of NLA, Keto, and NLA + Keto on LDF}

The effect of drugs (that inhibit PGs and NOS) and of the NO donor SNP on baseline LDF is shown in Fig. 3, where data are expressed as $\% \mathrm{CVC}_{\max }$. NLA $(10 \mathrm{mM})$ alone had no effect on LDF, whereas Keto $(10 \mathrm{mM})$ significantly increased LDF $(P<0.0025)$. The increase caused by Keto administration was significantly blunted $(P<0.01)$ by the coadministration of NLA (NLA + Keto) compared with Keto alone and was not different from its untreated baseline. SNP, a NO donor given along with NLA to suppress endogenous NOS, resulted in an increase in baseline that was similar to the increase observed with Keto. The dose of NLA was determined in pilot experiments (not shown) to be the lowest concentration of NLA that maximally reduced the NO-dependent vasodilation response to $10 \mathrm{mM}$ acetylcholine.

\section{Effects of NLA, Keto, NLA + Keto, and NLA + SNP on RH}

Representative graphs showing the effects of intradermal drug administration on the LDF response to RH are shown in Fig. 4. The response to RH before and after NLA is not significantly different (Fig. 4, top). Keto, however, increases baseline flow and maximum hyperemic flow, increases the AUC, and prolongs the falloff of the hyperemic blood flow (Fig. 4, 2nd panel). The increase in postischemic flow is largely attenuated by the coadministration of NLA + Keto compared with Keto alone (Fig. 4, bottom).

Figure 5 shows the effects of NLA, Keto, NLA + Keto, and NLA + SNP on peak RH before (untreated) and after (treated) drug treatment. In addition, the difference in treated peak RH flow from treated baseline RH flow is shown. Postdrug-treated peak flows were not different for NLA or NLA + Keto but were significantly increased by Keto or NLA + SNP $(P<$ 0.001 ). 
The values for time to peak flow, the calculated time constant, and the AUC describing the hyperemia following ischemia are shown in Table 1.

These parameters are unaffected by NLA or NLA + Keto. On the other hand, these parameters are all markedly increased by Keto or NLA $+\operatorname{SNP}(P<0.001)$ and were significantly attenuated by adding NLA to Keto $(P<0.01)$. Figure 6 compares percent changes between untreated and treated time to peak flow, AUC, and time constant for the decay of peak flow during RH and shows that the time to peak flow, AUC, and time constant are increased similarly for Keto and NLA + SNP. However, these parameters are unchanged for NLA or NLA + Keto.

Since we measured differences in baseline blood flow between conditions (i.e., between sites before and after drug administration), it is possible that the effects of Keto in altering RH responses are related to nonspecific elevation in skin blood flow. To test this possibility, we administered isoproterenol, a PG-independent vasodilator that has been previously shown to produce substantial vasodilation (10). We instrumented the legs of five additional subjects with three microdialysis probes each and then allowed time for recovery. We performed untreated RH and then administered $10 \mathrm{mM}$ Keto at a rate of $2 \mu \mathrm{l} / \mathrm{min}$ to one site and increasing concentrations of isoproterenol (starting at $50 \mu \mathrm{M}$ to a maximum of $150 \mu \mathrm{M}$ ) to two other sites until the elevation in baseline skin blood flow approximated the flow at the adjacent Keto site. RH was repeated. Under these conditions we found that isoproterenol and Keto produced similar peak RH flows but that the time to peak flow, the AUC, and the time constant of decay of peak flow were significantly greater $(P<0.001)$ for Keto compared with isoproterenol as shown in Fig. 7.

\section{DISCUSSION}

In the present studies we show that the nonspecific COX-1 and -2 inhibitor Keto, when delivered directly into the intra-dermal space, augments all aspects of the LDF response to RH. These include baseline flow, peak RH flow, the time to peak flow, the AUC, and the time constant for decay of peak RH flow. These effects are NOS dependent, and, with the coadminstration of the NOS inhibitor NLA, the potentiating effects of Keto were highly attenuated. Thus COX inhibition produces a NOS-dependent effect on RH. One interpretation of the data is that COX-dependent PGs or COX itself may reduce NOS activity and thus bioavailable NO. This is supported by our finding that combined NLA + SNP administration produces RH that is similar to RH produced by Keto. Alternatively, the differences could be due to a blunting effect of NOS on PG inhibition-induced hyperemia.

Administration of isoproterenol in a dose that produces a similar increase in baseline flow also increases peak RH flow but fails to change the AUC, the time constant of decay of hyperemia, and the time to peak hyperemia. This demonstrates that the effect of Keto on RH is not just due to an increase in baseline flow.

COX inhibition with Keto nonspecifically blocks both vasodilator PGs (e.g., prostacyclin) and vasoconstrictor PGs (e.g., thromboxanes) (23). The effects of Keto are not likely to be mediated by epoxyeicosatrienoic acid pathways believed to comprise potent EDHFs (14) 
because Keto exerts no influence on their synthesis. However, the specific effects of EDHF on skin blood flow have not been determined, and thus their role in the hyperemic response cannot be ruled out. On the one hand, since Keto resulted in increased cutaneous baseline flow, one possibility is that the inhibition of vasoconstrictor PGs contributes to the induced vasodilation. On the other hand, NOS inhibition with NLA combined with Keto effectively blunts excessive vasodilation observed with Keto alone, indicating a dependence of the Keto response on intact NOS and suggesting that COX products inhibit NOS. In this case COX inhibition disinhibits or unmasks NO dependence of RH.

To investigate this possibility further, we used the NO donor SNP combined with NLA to block endogenous NO production. We titrated the SNP dose to produce a similar increase in baseline LDF as was observed with Keto. We observed increases in the AUC, time constant, and time to peak flow that were similar to observations when using Keto alone. Increased bioactive NO during RH would therefore be unavailable under untreated conditions as reported by Zhao et al. (38) but may become available during COX inhibition.

The data suggest that Keto, a nonspecific COX inhibitor, increases bioavailable NO either through increased NOS activity or decreased NO destruction. Evidence for both hypotheses exists: NOS isoforms and COX interact (22), and thromboxane $\mathrm{A}_{2}$ exerts inhibitory effects on bioavailable NO through increasing oxidative stress (32) and inhibiting NOS (36).

Interactions between the biosynthesis, release, and effects of both NO and PGs have been reported in different tissues; in particular, increasing evidence suggests that there is considerable cross talk between NO and PG biosynthetic pathways involving an active back modulation operated by reaction end products, including NO, PGs, and cyclic nucleotides (22). However, the mechanisms of these interactions are often unclear, and the effect of NO and NO donors on PG biosynthesis and release still remains to be elucidated. It is thought that the interaction between NO and PG generation occurs at different levels. NO is thought to interfere directly with COX expression and PG biosynthesis. COX metabolites can interfere with NO biosynthesis. Furthermore, the expression of NOS is related to PG receptors and intracellular messengers, such as cAMP generated by activation of G-coupled PG receptors. In addition, both NO and PGs interact with their own respective biosynthetic pathway by modulating molecular events underlying NOS and COX expression (22).

\section{Comparison with the Literature}

We found that baseline LDF was significantly increased with Keto, which is similar to the data of Kellogg et al. (18), although they did not report significance at similar doses. This response was maintained over a 90-min time frame in our present study. Holowatz et al. (16) also showed a significant increase in forearm skin blood flow with Keto compared with control when studying aged human skin. This suggests that Keto may inhibit vasoconstrictive prostanoid production or disinhibit NOS as our data suggest. The lack of similarity between our study and other studies may be a result of inherent differences in the regulatory properties of forearm versus calf skin, similar to that shown for young and aged skin (16). 
These results contrast with those of Dalle-Ave et al. (12) who found that neither cutaneous RH nor the cutaneous hyperemic response to acetylcholine depended on PGs. Results also contrast with those of Binggeli et al. (3) who found that aspirin reduced rather than increased RH flow. Some of the differences of our study from these other studies may be due to our use of locally delivered Keto versus the use of systemically administered PG inhibitors in the other studies $(28,29)$. Systemic administration may exert potential generalized effects indirectly affecting peripheral blood flow. Our results are also different from those of Carlsson et al. (6) who studied the effects of PG inhibition with indomethacin in the cutaneous circulation of the finger and in the whole forearm (7), generally finding vasoconstriction rather than vasodilation in response to COX inhibition. The differences between results reported for indomethacin, aspirin, and Keto may be due to the relative effects of COX-1 and -2 inhibition and on the production of vasoconstrictive versus vasodilatory PGs.

Our results are consistent with the findings of Wong et al. (35) and Zhao et al. (38) in that the nonselective NOS inhibitor NLA exerted no effect on RH when given alone. However, we did observe powerful effects of NOS inhibition when it was combined with PG inhibition. This effect is appropriate given the interactions between NO and PG biosynthetic pathways involving an active back modulation operated by reaction end products, including NO, PGs, and cyclic nucleotides (22), or even from combined effects of COX metabolites and NO on EDHF $(2,25)$. In this regard, Nishikawa et al. (25), using coronary microvessels, demonstrated that the application of minute quantities of an NO donor could inhibit EDHF. However, in pilot experiments starting from nanomolar doses in NOS- and COX-blocked skin, we were unable to replicate these findings. An attempt by Schrage et al. (27) in human skeletal muscle also failed to show feedback inhibition from $\mathrm{NO}$ on the production of EDHF.

Our results might also be compared with the work of Engelke et al. (13) in the human forearm preparation. They found little effect of NOS inhibition alone on RH but potent interactions between a nonselective COX inhibitor (ibuprofen, given orally) and intraarterial administration of the nonisoform-selective NOS inhibitor $N^{\omega}$-monomethyl-L-arginine. These data, however, primarily reflect the response of skeletal muscle vasculature rather than skin that may account for directional differences in some results.

\section{Limitations}

Our observations are based on the response of calf skin to various physical and chemical perturbations. Most of the previous literature, however, has reported results of forearm skin blood flow. The response of these two tissues may not always be similar. Also, the conclusion that PGs and NO play a large role in RH responses in the skin is not the only possible explanation for the results.

The local control of cutaneous blood flow is more complex than the interplay among NO, PGs (both vasodilator and vasoconstrictor), and EDHF. Also, experiments seeking to inhibit the effects of EDHF have yet to be performed. However, the data support interactive contributions of $\mathrm{NO}$ and PGs in the regulation of postischemic (reactive hyperemic) skin blood flow. Interestingly, all of our interventions resulted in an increase of the parameters 
measured following RH or in no measurable change; none of the inhibitors tested reduced them. Thus the response to RH is likely controlled by local mediators as well as by the endogenous mechanical properties of the blood vessels.

In their article, Wong et al. (35) reported a significant amount of inter- and intrasubject variability even when RH was repeated during a single study day. This compared the response to ischemia in sites before and after microdialysis catheter insertion. They also compared an experimental, L-NAME-containing catheter with a control, and Ringer solution-containing catheter in the same subject. We also found that across-catheter responses are highly variable even if catheters are perfused with identical solutions. However, we found that repeated responses of a given single microdialysis site are much more reproducible since there is much less variability within a given catheter. An additional concern is that it is not possible to measure the drug concentration within the tissue tested; we only know that the drug concentrations in the perfusate and tissue levels are determined by transmembrane driving forces. We and others using similar techniques must therefore depend on the use of drug concentrations that have a demonstrable effect on the parameter being measured.

One additional concern is that of the phase of the menstrual cycle of the female subjects and the effects of altered hormonal status among subjects. Although this was not controlled for, since determinations were done on 1 or 2 sequential days, individual measurements of the response to RH were not likely to vary in each subject.

In summary, we have shown that the skin response to $\mathrm{RH}$ is enhanced by Keto administered locally using microdialysis, that the Keto potentiated RH was attenuated by NOS inhibition with NLA, and that the enhanced response to Keto alone is similar to the response to the NO donor SNP. Taken together, the data suggest that COX or COX products inhibit cutaneous NOS and that COX inhibition therefore unmasks NO dependence of cutaneous RH in humans.

\section{Acknowledgments}

We thank Dr. Leonard Newman and the Division of Pediatric Cardiology, especially, Dr. Michael H. Gewitz, for unflagging support. We also thank Drs. Thomas H. Hintze, David Robertson, and Phillip Low for constant inspiration and stimulation.

\section{GRANTS}

This work was supported National Institutes of Health Grants R01-HL-074873 and 1R21-DK-071647.

\section{References}

1. Anderson C, Andersson T, Wardell K. Changes in skin circulation after insertion of a microdialysis probe visualized by laser Doppler perfusion imaging. J Invest Dermatol. 1994; 102:807-811. [PubMed: 8176267]

2. Bauersachs J, Popp R, Hecker M, Sauer E, Fleming I, Busse R. Nitric oxide attenuates the release of endothelium-derived hyperpolarizing factor. Circulation. 1996; 94:3341-3347. [PubMed: 8989149]

3. Binggeli C, Spieker LE, Corti R, Sudano I, Stojanovic V, Hayoz D, Luscher TF, Noll G. Statins enhance postischemic hyperemia in the skin circulation of hypercholesterolemic patients: a 
monitoring test of endothelial dysfunction for clinical practice? J Am Coll Cardiol. 2003; 42:71-77. [PubMed: 12849662]

4. Bjornberg J, Albert U, Mellander S. Resistance responses in proximal arterial vessels, arterioles and veins during reactive hyperaemia in skeletal muscle and their underlying regulatory mechanisms. Acta Physiol Scand. 1990; 139:535-550. [PubMed: 2248033]

5. Buxton IL, Cheek DJ, Eckman D, Westfall DP, Sanders KM, Keef KD. $N^{\mathrm{G}}$-nitro-L-arginine methyl ester and other alkyl esters of arginine are muscarinic receptor antagonists. Circ Res. 1993; 72:387395. [PubMed: 7678206]

6. Carlsson I, Linde B, Wennmalm A. Arachidonic acid metabolism and regulation of blood flow: effect of indomethacin on cutaneous and subcutaneous reactive hyperaemia in humans. Clin Physiol. 1983; 3:365-373. [PubMed: 6684521]

7. Carlsson I, Sollevi A, Wennmalm A. The role of myogenic relaxation, adenosine and prostaglandins in human forearm reactive hyperaemia. J Physiol. 1987; 389:147-161. [PubMed: 3681724]

8. Chang HY, Chen CW, Hsiue TR. Comparative effects of L-NOARG and L-NAME on basal blood flow and ACh-induced vasodilatation in rat diaphragmatic microcirculation. Br J Pharmacol. 1997; 120:326-332. [PubMed: 9117127]

9. Costa F, Sulur P, Angel M, Cavalcante J, Haile V, Christman B, Biaggioni I. Intravascular source of adenosine during forearm ischemia in humans: implications for reactive hyperemia. Hypertension. 1999; 33:1453-1457. [PubMed: 10373232]

10. Crandall CG, Etzel RA, Johnson JM. Evidence of functional beta-adrenoceptors in the cutaneous vasculature. Am J Physiol Heart Circ Physiol. 1997; 273:H1038-H1043.

11. Dakak N, Husain S, Mulcahy D, Andrews NP, Panza JA, Waclawiw M, Schenke W, Quyyumi AA. Contribution of nitric oxide to reactive hyperemia: impact of endothelial dysfunction. Hypertension. 1998; 32:9-15. [PubMed: 9674631]

12. Dalle-Ave A, Kubli S, Golay S, Delachaux A, Liaudet L, Waeber B, Feihl F. Acetylcholineinduced vasodilation and reactive hyperemia are not affected by acute cyclooxygenase inhibition in human skin. Microcirculation. 2004; 11:327-336. [PubMed: 15280072]

13. Engelke KA, Halliwill JR, Proctor DN, Dietz NM, Joyner MJ. Contribution of nitric oxide and prostaglandins to reactive hyperemia in human forearm. J Appl Physiol. 1996; 81:1807-1814. [PubMed: 8904603]

14. Fleming I, Busse R. Endothelium-derived epoxyeicosatrienoic acids and vascular function. Hypertension. 2006; 47:629-633. [PubMed: 16490839]

15. Hintze TH, Vatner SF. Reactive dilation of large coronary arteries in conscious dogs. Circ Res. 1984; 54:50-57. [PubMed: 6692499]

16. Holowatz LA, Thompson CS, Minson CT, Kenney WL. Mechanisms of acetylcholine-mediated vasodilatation in young and aged human skin. J Physiol. 2005; 563:965-973. [PubMed: 15661816]

17. Johnsson E, Folkow B, Karlstrom G. Myogenic responsiveness in rat hindquarter vessels during constant-flow and constant-pressure perfusion in vitro; effects of various potassium concentrations and of endothelial nitrous oxide blockade. Acta Physiol Scand. 1991; 142:319-328. [PubMed: 1927547]

18. Kellogg DL Jr, Zhao JL, Coey U, Green JV. Acetylcholine-induced vasodilation is mediated by nitric oxide and prostaglandins in human skin. J Appl Physiol. 2005; 98:629-632. [PubMed: 15649880]

19. Kristensen JK, Henriksen O. Excess cumulative blood flow and repayment during reactive hyperemia in human cutaneous tissue. Acta Physiol Scand. 1980; 108:1-6. [PubMed: 7376901]

20. Larkin SW, Williams TJ. Evidence for sensory nerve involvement in cutaneous reactive hyperemia in humans. Circ Res. 1993; 73:147-154. [PubMed: 8508526]

21. McCord GR, Cracowski JL, Minson CT. Prostanoids contribute to cutaneous active vasodilation in humans. Am J Physiol Regul Integr Comp Physiol. 2006; 291:R596-R602. [PubMed: 16484440]

22. Mollace V, Muscoli C, Masini E, Cuzzocrea S, Salvemini D. Modulation of prostaglandin biosynthesis by nitric oxide and nitric oxide donors. Pharmacol Rev. 2005; 57:217-252. [PubMed: 15914468]

23. Moncada S, Vane JR. Pharmacology and endogenous roles of prostaglandin endoperoxides, thromboxane $\mathrm{A}_{2}$, and prostacyclin. Pharmacol Rev. 1978; 30:293-331. [PubMed: 116251] 
24. Monsuez JJ. Mediators of reactive hyperemia. [In French]. Arch Mal Coeur Vaiss. 2001; 94:591599. [PubMed: 11480157]

25. Nishikawa Y, Stepp DW, Chilian WM. Nitric oxide exerts feedback inhibition on EDHF-induced coronary arteriolar dilation in vivo. Am J Physiol Heart Circ Physiol. 2000; 279:H459-H465. [PubMed: 10924042]

26. Press, WH.; Teukolsky, WT.; Vetterling, SA.; Flannery, BP. Numerical Recipes in C. Cambridge, UK: Cambridge University Press; 1992. p. 59-70.

27. Schrage WG, Dietz NM, Eisenach JH, Joyner MJ. Agonist-dependent variablity of contributions of nitric oxide and prostaglandins in human skeletal muscle. J Appl Physiol. 2005; 98:1251-1257. [PubMed: 15563630]

28. Shastry S, Dietz NM, Halliwill JR, Reed AS, Joyner MJ. Effects of nitric oxide synthase inhibition on cutaneous vasodilation during body heating in humans. J Appl Physiol. 1998; 85:830-834. [PubMed: 9729554]

29. Shastry S, Minson CT, Wilson SA, Dietz NM, Joyner MJ. Effects of atropine and L-NAME on cutaneous blood flow during body heating in humans. J Appl Physiol. 2000; 88:467-472. [PubMed: 10658012]

30. Shepherd JT. Reactive hyperemia in human extremities. Circ Res. 1964; 15(Suppl):76-79. [PubMed: 14206323]

31. Stewart J, Kohen A, Brouder D, Rahim F, Adler S, Garrick R, Goligorsky MS. Noninvasive interrogation of microvasculature for signs of endothelial dysfunction in patients with chronic renal failure. Am J Physiol Heart Circ Physiol. 2004; 287:H2687-H2696. [PubMed: 15297253]

32. Tang M, Cyrus T, Yao Y, Vocun L, Pratico D. Involvement of thromboxane receptor in the proatherogenic effect of isoprostane F2alpha-III: evidence from apolipoprotein E- and LDL receptor-deficient mice. Circulation. 2005; 112:2867-2874. [PubMed: 16267259]

33. Tur E, Yosipovitch G, Bar-On Y. Skin reactive hyperemia in diabetic patients. A study by laser Doppler flowmetry. Diabetes Care. 1991; 14:958-962. [PubMed: 1797508]

34. Vuilleumier P, Decosterd D, Maillard M, Burnier M, Hayoz D. Postischemic forearm skin reactive hyperemia is related to cardovascular risk factors in a healthy female population. J Hypertens. 2002; 20:1753-1757. [PubMed: 12195115]

35. Wong BJ, Wilkins BW, Holowatz LA, Minson CT. Nitric oxide synthase inhibition does not alter the reactive hyperemic response in the cutaneous circulation. J Appl Physiol. 2003; 95:504-510. [PubMed: 12692141]

36. Yamada T, Fujino T, Yuhki K, Hara A, Karibe H, Takahata O, Okada Y, Xiao CY, Takayama K, Kuriyama S, Taniguchi T, Shiokoshi T, Ohsaki Y, Kikuchi K, Narumiya S, Ushikubi F. Thromboxane $\mathrm{A}_{2}$ regulates vascular tone via its inhibitory effect on the expression of inducible nitric oxide synthase. Circulation. 2003; 108:2381-2386. [PubMed: 14557367]

37. Zatta AJ, Headrick JP. Mediators of coronary reactive hyperaemia in isolated mouse heart. Br J Pharmacol. 2005; 144:576-587. [PubMed: 15655499]

38. Zhao JL, Pergola PE, Roman LJ, Kellogg DL Jr. Bioactive nitric oxide concentration does not increase during reactive hyperemia in human skin. J Appl Physiol. 2004; 96:628-632. [PubMed: $14715681]$ 


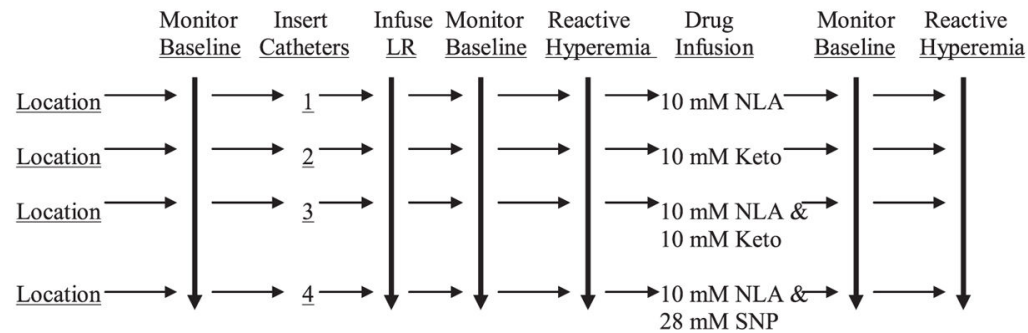

Fig. 1.

Scheme for the described studies. First, locations on the leg were randomly designated 1-4, and baseline laser-Doppler flow was measured. Microdialysis catheters were then inserted and perfused with vehicle [sterile lactated Ringer solution (LR)], and laser-Doppler flow was monitored until levels returned to preinsertion values. An initial reactive hyperemia (RH) was performed, and after return of laser Doppler to pre-RH values, drugs were infused for at least $30 \mathrm{~min}$ while laser-Doppler flow was monitored. RH was repeated once again while the indicated drug was perfused. NLA, nitro-L-arginine; Keto, ketorolac; SNP, sodium nitroprusside. 


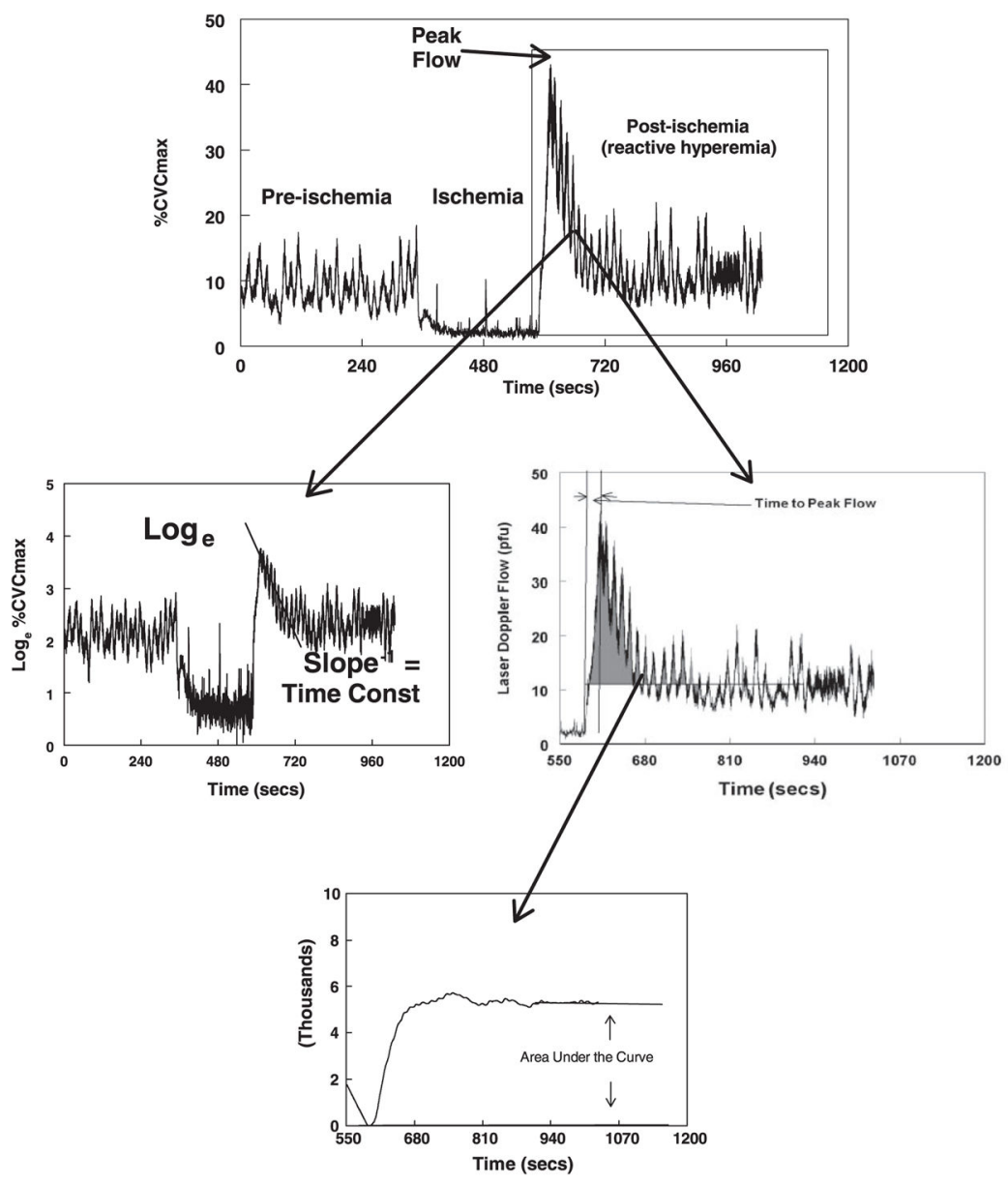

Fig. 2.

Top: representative $\mathrm{RH}$ tracing during measurement of laser-Doppler flow expressed as maximum percent cutaneous vascular conduction $\left(\% \mathrm{CVC}_{\max }\right)$ where $\mathrm{CVC}$ is laser-Doppler flow/mean arterial pressure. This is divided into preischemia, ischemia, and postischemia phases. Middle, left: an approximately exponential falloff of hyperemic flow; the time constant (Const) of this falloff is analyzed by logarithmic transformation and linear least squares fit. Middle, right: postischemia phase. Area under the curve (AUC) is shown as the grayed area, and the calculation (bottom) is obtained by taking the difference between the steady-state amplitude of this integral and its value at the onset of hyperemia (i.e., at occlusion cuff release). pfu, Perfusion unit. 

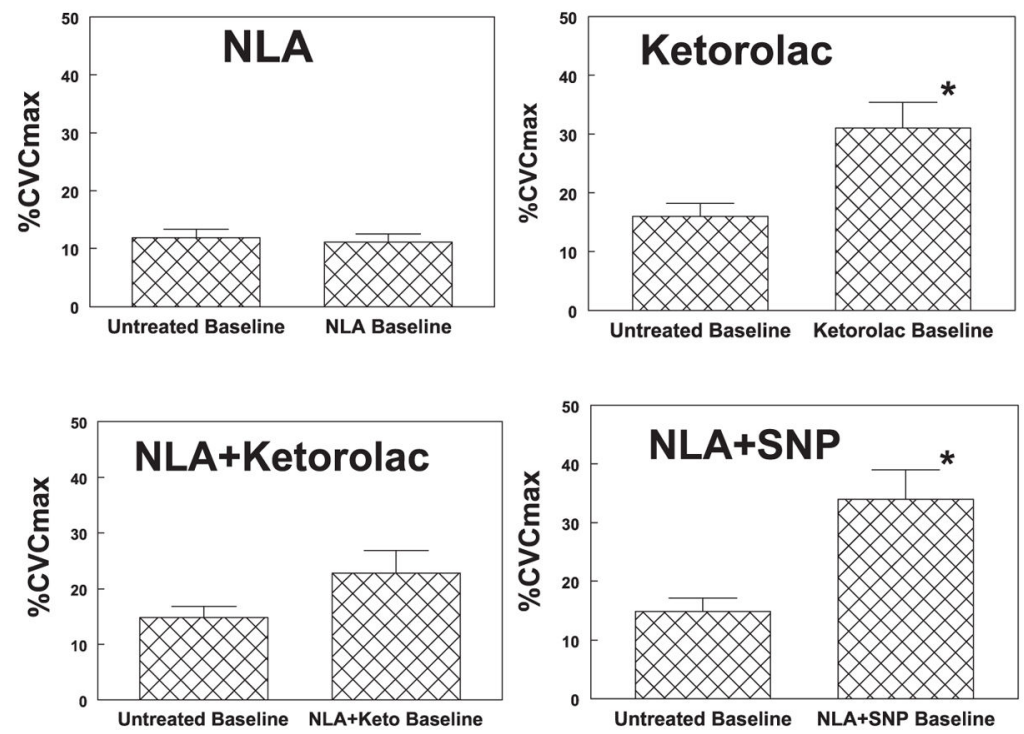

Fig. 3.

The $\% \mathrm{CVC}_{\max }$ before and after the administration of NLA, Keto, NLA + Keto, and NLA + SNP. These comprise the "treated baseline." Laser-Doppler flow is expressed as $\% \mathrm{CVC}_{\max }$, where CVC is laser-Doppler flow/mean arterial pressure. Keto increased baseline blood conductance. This increase was blunted by combining NLA with Keto. SNP was given in a dose that increased CVC by an amount similar to the increase produced by Keto. $* P<0.05$ compared with baseline. 

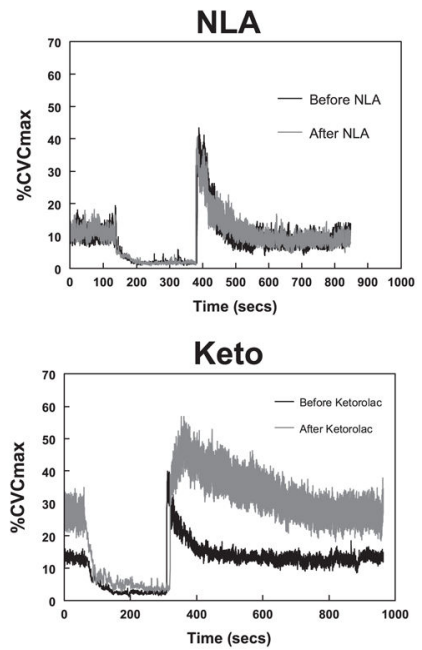

NLA+Keto

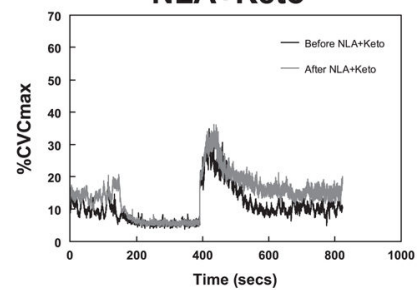

NLA+SNP

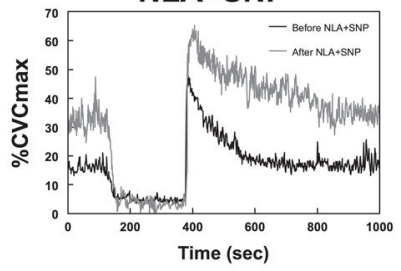

Fig. 4.

Representative RH responses to NLA alone (top), Keto alone (second panel), combined NLA + Keto (third panel), and combined NLA + SNP (bottom). All tracings are from a single subject. RH responses before drugs are shown in black. RH responses after drugs are shown in gray. NLA alone does not affect RH. Keto or NLA + SNP increases the magnitude and AUC of RH, whereas its combination with NLA (i.e., NLA + Keto) attenuates the potentiating effects of Keto. 


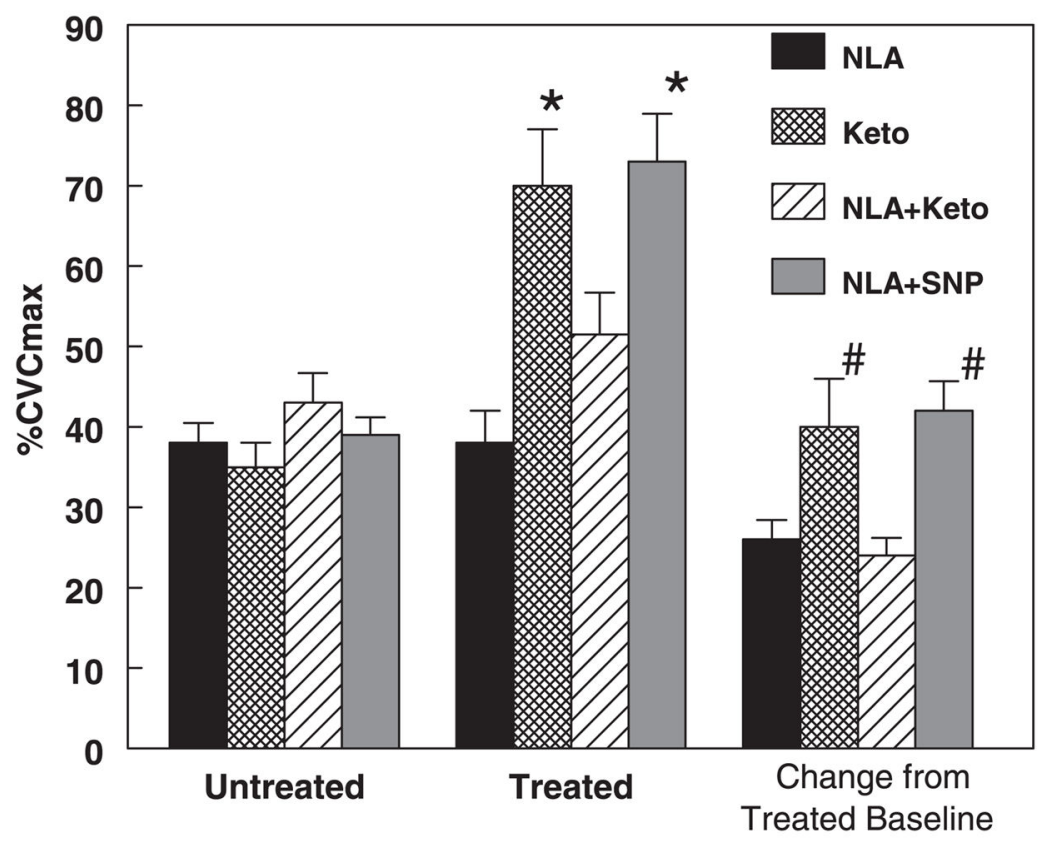

Fig. 5.

Peak RH flows before drugs (untreated, left bars) after receiving drugs (treated, middle bars), and the difference between the peak flow while treated and the baseline flow while treated averaged over all subjects (change from treated baseline, right bars). Laser-Doppler flow is expressed as $\% \mathrm{CVC}_{\max }$, where $\mathrm{CVC}$ is laser-Doppler flow/mean arterial pressure. Peak flow is increased by Keto and by NLA + SNP compared with untreated peak flow. The response to Keto alone is attenuated by the addition of NLA (NLA + Keto). The increase of peak flow above the treated baseline is larger for Keto alone and the combination of NLA + SNP compared with NLA or NLA + Keto. ${ }^{*} P<0.05$ compared with untreated; $\# P<0.05$ compared with NLA. 


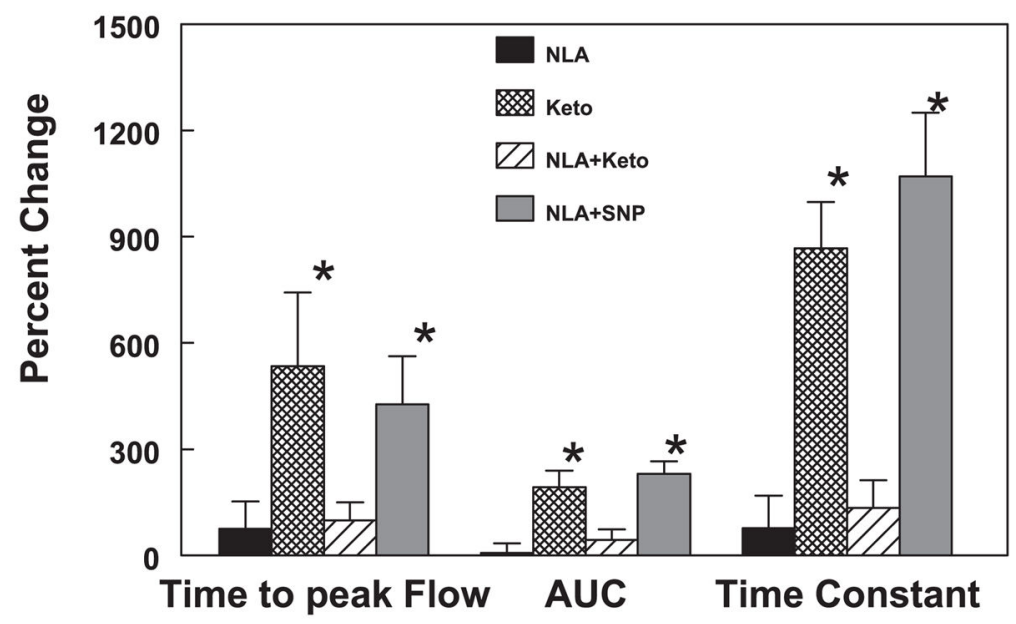

Fig. 6.

Percent changes in time to peak flow (left bars), the AUC (middle bars), and the time constant of decay from peak flow (right bars) with drug treatment averaged over all subjects. Time to peak flow, AUC, and time constant are increased for Keto and NLA + SNP but not for NLA or NLA + Keto. ${ }^{*} P<0.05$ compared with untreated control. 

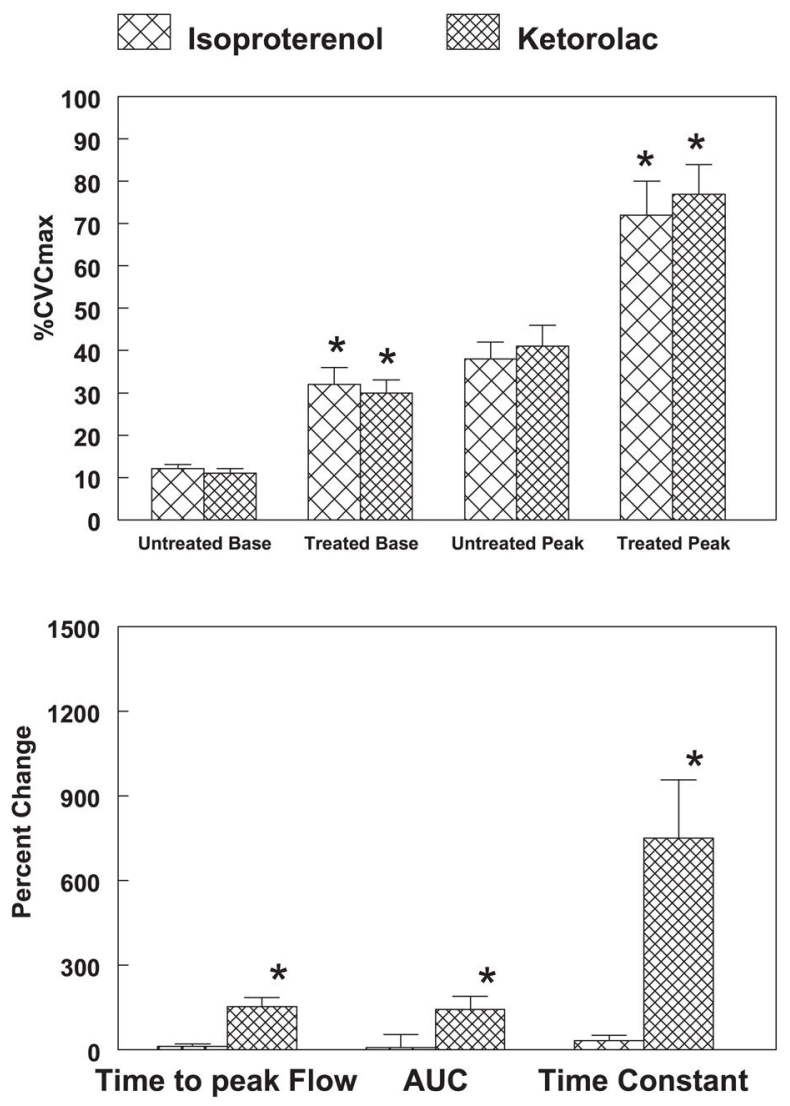

Fig. 7.

Top: baseline flows (Base) and peak RH flows before (untreated) and after (treated) receiving isoproterenol or Keto. Laser-Doppler flow is expressed as $\% \mathrm{CVC}_{\max }$, where CVC is laser-Doppler flow/mean arterial pressure. Bottom: percent changes in time to peak flow (left bars), the AUC (middle bars), and the time constant of decay from peak flow (right bars) with isoproterenol or Keto treatment. Time to peak flow, AUC, and time constant are increased for Keto but not for isoproterenol. ${ }^{*} P<0.05$ compared with untreated. 

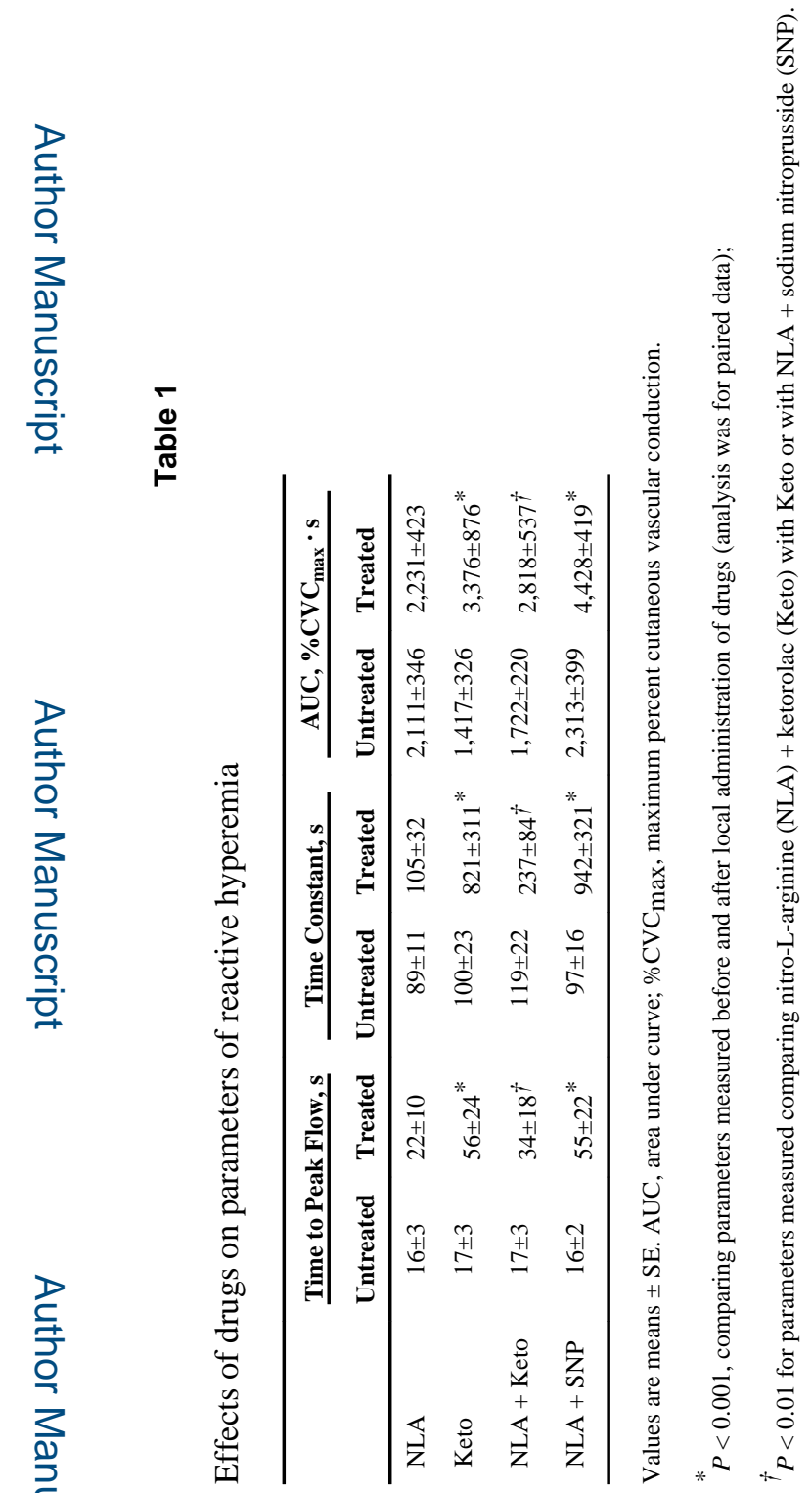

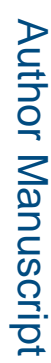

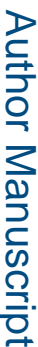

Am J Physiol Heart Circ Physiol. Author manuscript; available in PMC 2015 July 22. 